\title{
The Existence of Homoclinic Solutions for Second Order Hamiltonian System
}

\author{
Jie Gao
}

School of Mathematics and Information Sciences, Weifang University, Weifang, Shandong 261061, China

\begin{abstract}
The research of homoclinic orbits for Hamiltonian system is a classical problem, it has valuable applications in celestial mechanics, plasma physis, and biological engineering. For example, homoclinic orbits rupture can yield chaos lead to more complex dynamics behaviour. This paper studies the existence of homoclinic solutions for a class of second order Hamiltonian system, we will prove this system exists at least one nontrivial homoclinic solution.
\end{abstract}

Index Terms: Homoclinic solution; Hamiltonian system; critical point; （PS） condition

(C) 2011 Published by MECS Publisher. Selection and/or peer review under responsibility of the Research Association of Modern Education and Computer Science

\section{Introduction}

The existence of homoclinic solutions for Hamiltonian systems and their importance in the study of the behavior of dynamical systems have been already recognized from Poincaré[1]. In the past decade, this problem has been studied intensively by many mathematicians. Many authors have studied the existence of homoclinic solutions for Hamiltonian systems via critical point theory and variational methods, see[2]-[5] and references therein.

The purpose of this paper is to study the existence of homoclinic solutions for second order Hamiltonian system

$$
\ddot{u}-A(t) u+V_{u}(t, u)=0 .
$$

Wher $t \in R, u \in R^{n}, V_{u}(t, u)$ denotes the gradient of $V(t, u)$ with respect to $u$.

Definition 1.1 If the solution $u(t) \in C^{2}\left(R, R^{n}\right)$ of system (1.1) satisfies boundary value condition

$$
u( \pm \infty)=\dot{u}( \pm \infty)=0 .
$$

* Corresponding author.

E-mail address: gaojie71@126.com 
Then $u(t)$ is called the homoclinic solution (to 0 ) of (1.1). If $u(t) \neq 0$, then $u(t)$ is called the nontrivial homoclinic solution .

Definition 1.2 $I \in C^{1}(B, R)$ is said to satisfiy the (PS) condition if any sequence $\left\{u_{j}\right\}_{j \in N} \subset B$ for which $\left\{I\left(u_{j}\right)\right\}_{j \in N}$

Is bounded and $I^{\prime}\left(u_{j}\right) \rightarrow 0$ as $j \rightarrow+\infty$, possesses a convergent subquence in $B$.

To state the main result, we state the basic conditions on $A(t)$ and $V(t, u)$ :

(I) $A \in C\left(R, R^{n^{2}}\right)$ is a symmetric and positive definite matrix for all $t \in R$; there exists a continuous function $f: R \rightarrow R$ such that

$$
(A(t) u, u) \geq f(t)|u|^{2}, \quad \forall(t, u) \in R \times R^{n} .
$$

where $f$ satisfies: $f(t)>0$ for all $t \in R$, and $f(t) \rightarrow+\infty$ as $|t| \rightarrow+\infty$.

From this condition, we see that there exists a constant $b>0$ such that

$(A(t) u, u) \geq b|u|^{2}, \quad \forall(t, u) \in R \times R^{n}$.

(II) $V(t, u) \geq 0$ for all $(t, u) \in R \times R^{n}$ and there exists constants $K>0$ and $M_{1}>0$ such that

$$
V(t, u) \leq K|u|^{2},(t, u) \in R \times R^{n},|u| \leq M_{1},
$$

where $2 K<b$, with $b$ is defined in (1.2).

(III) there exists function $f_{0}: R \rightarrow R^{+}$, constant $c_{1}>2$ and $M_{2}>0$ such that

$$
V(t, u) \geq f_{0}(t)|u|^{c_{1}}, \forall(t, u) \in R \times R^{n},|u| \geq M_{2} .
$$

(IV) there exists constants $l>2$ and $c_{2}$ with $0 \leq c_{2}<\frac{l-2}{2}$,

such that

$$
l V(t, u)-\left(V_{u}(t, u), u\right) \leq c_{2}(A(t) u, u), \forall(t, u) \in R \times R^{n} .
$$

(V) $V_{u}(t, u)=O(|u|)$ as $|u| \rightarrow 0$ uniformly with respect to $t \in R$.

(VI) there exists $W \in C\left(R^{n}, R\right)$ such that

$$
\left|V_{u}(t, u)\right| \leq|W(u)|, \quad \forall(t, u) \in R \times R^{n} .
$$

\section{Preliminary knowledges}

We donote $H^{1}\left(R, R^{n}\right)$ and $L^{p}\left(R, R^{n}\right)(2 \leq p<+\infty)$ as the Banach space of functions on $R$ with value in $R^{n}$ under the norms 


$$
\|u\|_{H^{1}}:=\left(\|u\|_{2}^{2}+\|\dot{u}\|_{2}^{2}\right)^{\frac{1}{2}} ; \quad\|u\|_{p}:=\left(\int_{R}|u(t)|^{p} d t\right)^{\frac{1}{p}}
$$

Respectively.

Let

$$
E=\left\{u \in H^{1}\left(R, R^{n}\right): \int_{R}\left[|\dot{u}(t)|^{2}+(A(t) u(t), u(t))\right] d t<+\infty\right\},
$$

then $E$ is a Hilbert space with the inner product

$$
(x, y)=\int_{R}[(\dot{x}(t), \dot{y}(t))+(A(t) x(t), y(t))] d t,
$$

and the corresponding norm is $\|x\|^{2}=(x, x)$. Note that for $p \in[2$

$, \infty), E \subset H^{1}\left(R, R^{n}\right) \subset L^{p}\left(R, R^{n}\right)$ is continuous embedding.

In particular, for $p=+\infty$, there exists a constant $C>0$ such that $\|u\|_{\infty} \leq C\|u\|, \forall u \in E$.

Lemma 2.1[6] Suppose that $A(t)$ satisfies condition (I), then $E$ embedding into $L^{2}\left(R, R^{n}\right)$ is compact.

Lemma 2.2[6] Suppose that conditions (I),(V),(VI) are satisfied . If $u_{k}$ weakly convergence to $u_{0}$ in $E$, then $V_{u}\left(t, u_{k}\right)$ strongly convergence to $V_{u}\left(t, u_{0}\right)$ in $L^{2}\left(R, R^{n}\right)$.

Now we difine the functional $I: E \rightarrow R$ as follows:

$$
\begin{aligned}
I(u) & =\int_{R}\left[\frac{1}{2}|\dot{u}(t)|^{2}+\frac{1}{2}(A(t) u(t), u(t))-V(t, u(t))\right] d t \\
& =\frac{1}{2}\|u\|^{2}-\int_{R} V(t, u(t)) d t .
\end{aligned}
$$

Lemma 2.3[7] Suppose that conditions (I)-(VI) are satisfied, then for all $u, v \in E$, we have

$$
I^{\prime}(u) v=\int_{R}\left[(\dot{u}(t), \dot{v}(t))+(A(t) u(t), v(t))-\left(V_{u}(t, u(t)), v(t)\right)\right] d t .
$$

From this we obtain

$$
I^{\prime}(u) u=\|u\|^{2}-\int_{R}\left(V_{u}(t, u(t)), u(t)\right) d t .
$$

Moreover, $I$ is a continuously Fréchet-differentiable functional defined on $E$, namely, $I \in C^{1}(E, R)$ and any critical point $u$ of $I$ on $E$ is a classical solution of (1.1) with $u( \pm \infty)=0=\dot{u}( \pm \infty)$.

Lemma 2.4 Suppose that conditions (I),(IV),(V),(VI) are satisfied, then $I$ satisfies the (PS) condition.

Proof Assume that $\left\{u_{j}\right\}_{j \in N} \subset E$ is a sequence such that $\left\{I\left(u_{j}\right)\right\}_{j \in N}$ is bounded and $I^{\prime}\left(u_{j}\right) \rightarrow 0$ as $j \rightarrow+\infty$. Then there has a constant $M>0$ such thatt

$\left|I\left(u_{j}\right)\right| \leq M, \quad\left\|I^{\prime}\left(u_{j}\right)\right\|_{E^{*}} \leq M, \quad j \in N$

By (2.1),(2.3) and condition (IV), we have 


$$
\begin{aligned}
\left(\frac{l}{2}-1\right)\left\|u_{j}\right\|^{2}= & I I\left(u_{j}\right)-I^{\prime}\left(u_{j}\right) u_{j} \\
& +\int_{R}\left(l V\left(t, u_{j}(t)\right)-\left(V_{u}\left(t, u_{j}(t)\right), u_{j}(t)\right)\right) d t \\
& \leq l I\left(u_{j}\right)-I^{\prime}\left(u_{j}\right) u_{j}+c_{2} \int_{R}\left(A(t) u_{j}(t), u_{j}(t)\right) d t
\end{aligned}
$$

Define

$$
q(u)=\int_{R}\left[\left(\frac{l-2}{2}\right)|\dot{u}(t)|^{2}+\left(\frac{l-2}{2}-c_{2}\right)(A(t) u(t), u(t))\right] d t,
$$

then we have

$$
l_{1}\|u\|^{2} \leq q(u) \leq l_{2}\|u\|^{2},
$$

where $l_{1}=\frac{l-2}{2}-c_{2}, l_{2}=\frac{l-2}{2}$. Thus, by (2.4),(2.5) and (2.6),

we obtain that

$$
l_{1}\left\|u_{j}\right\|^{2} \leq q\left(u_{j}\right) \leq l I\left(u_{j}\right)-I^{\prime}\left(u_{j}\right) u_{j} \leq l M+M\left\|u_{j}\right\| .
$$

Since $l_{1}>0$, then (2.7) shows that $\left\{u_{j}\right\}_{j \in N}$ is bounded in $E$. By lemma 2.1, the sequence $\left\{u_{j}\right\}_{j \in N}$ has a subsequence, again denoted by $\left\{u_{j}\right\}_{j \in N}$, and there exists $u \in E$ such that $u_{j}$ weakly convergence to $u$ in $E, u_{j}$ strongly convergence to $u$ in $L^{2}\left(R, R^{n}\right)$.

Thus

$$
\left(I^{\prime}\left(u_{j}\right)-I^{\prime}(u)\right)\left(u_{j}-u\right) \rightarrow 0 .
$$

By lemma 2.2 and Hölder inequality, we have

$$
\int_{R}\left(V_{u}\left(t, u_{j}(t)\right)-V_{u}(t, u(t)), u_{j}(t)-u(t)\right) d t \rightarrow 0 .
$$

if $j \rightarrow+\infty$. On the other hand, an easy computation shows that

$$
\begin{aligned}
& \left(I^{\prime}\left(u_{j}\right)-I^{\prime}(u), u_{j}-u\right) \\
& =\left\|u_{j}-u\right\|^{2}-\int_{R}\left(V_{u}\left(t, u_{j}(t)\right)-V_{u}(t, u(t)), u_{j}(t)-u(t)\right) d t .
\end{aligned}
$$

Hence, $\left\|u_{j}-u\right\| \rightarrow 0$ as $j \rightarrow+\infty$. Namely, I satisfies the (PS) condition.

Lemma 2.5[8] Let $B$ is a real Banach space and $I \in C^{1}(B, R)$ satisfying the (PS) condition, suppose that $I(0)=0$ and

$\left(\mathrm{H}_{1}\right)$ there exists constants $\rho, a>0$ such that $\left.I\right|_{\partial B_{\rho}} \geq a$;

$\left(\mathrm{H}_{2}\right)$ there exists $e \in B \backslash \bar{B}_{\rho}$ such that $I(e) \leq 0$.

Then $I$ possesses a critical value $c \geq a>0$ given by 


$$
c=\inf _{g \in \Gamma \in[0,1]} \max _{s \in[1} I(g(s))
$$

Where

$$
\Gamma=\{g \in C([0,1], B): g(0)=0, g(1)=e\}
$$

\section{Main Result}

Theorem 3.1 Suppose that conditions (I)-(VI) are satisfied, then the Hamiltonian system (1.1) possesses at least one nontrivial homoclinic solution.

Proof By condition (II), we know $I(0)=0$. On the other hand, by lemma 2.3 and 2.4, we know $I \in C^{1}(E, R)$ satisfies the (PS) condition.

Assume that $u \in E, 0<\|u\|_{\infty} \leq M_{1}$, then by (1.2)and condition (II), we have

$$
\int_{R} V(t, u(t)) d t \leq K \int_{R}|u(t)|^{2} d t \leq K\|u\|_{2}^{2} \leq \frac{K}{b}\|u\|^{2} .
$$

Combining (2.1), we have

$$
I(u) \geq \frac{1}{2}\|u\|^{2}-\frac{K}{b}\|u\|^{2}=\frac{1}{2}\left(1-\frac{2 K}{b}\right)\|u\|^{2} .
$$

Condition (II) implies $1-\frac{2 K}{b}>0$. Let

$$
\rho=\frac{M_{1}}{C}>0 ; \quad a=\frac{(b-2 K) M_{1}^{2}}{2 b C^{2}}>0 .
$$

Then there exists $\rho>0$ and $a>0$ such that $\left.I\right|_{\partial B_{\rho}} \geq a$ (by (2.8). Namely, $I$ satisfies the condition $\left(\mathrm{H}_{1}\right)$ of lemma 2.5 .

By (2.1), for every $m \in R \backslash\{0\}$ and $u \in E \backslash\{0\}$, we have

$$
I(m u)=\frac{m^{2}}{2}\|u\|^{2}-\int_{R} V(t, m u(t)) d t .
$$

Take some $U \in E$ such that $\|U\|=1$. Then there exists a subset $\Omega$ of positive measure of $R$ shuch that $U(t) \neq 0$ for $t \in \Omega$. Let $m>0$ such that $m|U(t)| \geq M_{2}$ for $t \in \Omega$. Then by conditions (II) and (III), we have

$$
I(m U) \leq \frac{m^{2}}{2}-m^{c_{1}} \int_{\Omega} f_{0}(t)|U(t)|^{c_{1}} d t .
$$

Since $f_{0}(t)>0, c_{1}>2$, then (2.10) implies that $I(m U)<0$ for some $m>0$ such that $m|U(t)| \geq M_{2}$ for $t \in \Omega$ and $\|m U\|>\rho$, where $\rho$ is defined in (2.9). Namely, $I$ satisfies the condition $\left(\mathrm{H}_{2}\right)$ of lemma 2.5. Then by lemma 2.5 , I possesses a critical value $c \geq a>0$ given by

$$
c=\inf _{g \in \Gamma \in[0,1]} \max _{s \in[} I(g(s)) .
$$


Where

$$
\Gamma=\{g \in C([0,1], E): g(0)=0, g(1)=m U\} .
$$

Hence, there exists $u \in E$ such that

$$
I(u)=c, \quad I^{\prime}(u)=0 .
$$

By lemma 2.3, hamiltonian system (1.1) possesses at least one nontrivial homoclinic solution. So we finish the proof of this theorem.

\section{References}

[1] H. Poincaré, "les méthodes nouvelles de la mécanique céleste", Gauthier-villars, pairs, pp.1897-1899 .

[2] E. Paturel, "Multiple homoclinic orbits for a class of Hamiltonian systems", Calc. Val. Partial Differential Equations, 12(2001),no.2, pp.117-143

[3] A. Flavia, "Periodic and homoclinic solutions to a class of Hamiltonian systems with indefinite potential in sign”, Boll.Un. Mat. Ital., B(7)10(1996), no.2, pp.303-324.

[4] M. Izydorek and J. Junczewska, "Homoclinic solutions for a class of the second order hamiltonian systems", J. Differential Equations, 219(2005), no.2, pp.375-389.

[5] Q. Zhang and C. Liu, "Infinitely many homoclinic solutions for second order Hamiltonian systems", Nonlinear Anal., 72(2010),pp.894-903.

[6] W. Omana and M. Willem, "Homoclinic orbits for a class of Hamiltonian systems", Differential Integral Equations, 5(1992), no.5, pp.1115-1120.

[7] Z. Zhang and R. Yuan, "Homoclinic solutions for a class of non-autonomous subquadratic second-order Hamiltonian systems", Nonlinear Analysis, 71(2009), pp.4125-4130.

[8] P. H. Rabinowitz, "Minimax Methods in Critical Point Theory with Applications to Differential Equations", CBMS Reg.Conf. Ser. in. Math., vol. 65, America Mathematical society, Provodence, RI, 1986 